\title{
Pt Nanoparticles as Electrocatalysts for Water Splitting: Atomistic Insights into Function and Stability.
}

\author{
R. Schlögl ${ }^{1,3}$, E. Kudrenko ${ }^{1}$, M. Willinger ${ }^{1}, Y$. Yi ${ }^{1}$, R. Arrigo ${ }^{3}$, F. Schüth ${ }^{2}$
}

1. Dept. Inorganic Chemistry, Fritz-Haber-Institut der Max-Planck-Gesellschaft, Berlin, Germany

2. Dept. Heterogeneous Catalysis, Max-Planck-Institut für Kohlenforschung, Mülheim/Ruhr, Germany

3. Max-Planck-Institut für chemische Energiekonversion (CEC), Mülheim/Ruhr, Germany

The grid-scale utilization of renewable energy requires additional measures to integrate fluctuating primary electricity into the energy demand structure. Moreover, buffers for long-term fluctuations of solar energy need to be installed in grid-load capacities when no fossil back-up structures should be used. Finally, a large part of our energy demand is bound to material energy carriers rather than to electricity. For all these reasons an interface between electrical energy and chemical energy[1] is of strategic relevance in future efficient and sustainable energy system.

Water splitting by (photo) electrical energy is at the center stage of chemical energy storage as it can provide the necessary dimensions of energy storage capacity and conceptually it can operate in sustainable modes. Electrolysis is a base technology with a long research history[2] but little practical application. The energy losses and electrode stability issues even at constant operation of the oxygen evolution side of the reaction are identified as the core obstacle. We have designed a reference model system of Pt nanoparticles on custom-designed mesoporous carbon structures[3] allowing an in-depth analysis of the mode of operation in fuel cell and water splitting applications. The system itself is of remarkable stability allowing meaningful analytical studies and micro analytical investigations with sufficient transferability between the local and integral dimensional scales.

The synthesis procedure leads to homogeneous Pt nanoparticles with dimensions of about $2 \mathrm{~nm}$. Thermal annealing to $1000 \mathrm{~K}$ leads to limited sintering and to substantial structural ordering transferring the rough initial particles into well-structured facetted nanoparticles.

Electrochemical cycling under mild conditions for $2 \mathrm{~h}$ reveals some clear trends: well-ordered particles are much better in hydrogen evolution than in oxygen evolution. Under OER conditions the ordering is rapidly lost. Both systems with and without thermal annealing undergo substantial structural degradation without macroscopic loss of Pt due to the carbon encapsulation.

HAADF-STEM analysis reveals that the degradation mechanism operates indeed via structural roughening and gradual dissolution. The dissolved Pt species re-precipitate as clusters or atomic species on the bare carbon surface.

The process was also followed by in-situ NAP XPS on model Pt nanoparticles supported on a NAFION membrane. These studies revealed the chemical sequence of events upon oxidative dissolution of Pt in water. These results are well consistent with the microscopic observations. The results were compared to the degradation of a massive Pt electrode. Both chemistry and nanostructruring were found to be very similar in extent and dimensions to the degradation of the surface of the massive metal converting itself into Pt-black. The electrochemically well-known ill-defined material turns out to be a mixture of 
hydrated Pt oxides converting itself upon storage in air into a thin film of Pt nanoparticles with sizes also below $5 \mathrm{~nm}$.

The multiple role of the carbon support was investigated. Carbon serves as holding and stabilizing agent for the Pt nanoparticles as well as it provides the electrified interface through contacts between the nanostructure and the macroscopic current collector. Conventional electrochemical carbon grades are of considerable structural variety when analyzed at the mesoscopic level. This emerges from the thermal pre-treatment of a still reactive carbon precursor. Hence it is difficult to derive structure-function relations with such substrates. The mesosphere carbon support employed here is of much better structural homogeneity allowing a correlation between the local structure and its binding capacity for Pt. The combination of TEM and EELS revealed clearly that an inverse correlation exists between the degree of graphitic ordering and the binding site density for Pt. As not unexpectedly the disordered carbon is sensitive to electro-corrosion it becomes apparent that the optimization of the support will be a critical task in finding an optimized electrocatalyst for OER applications in water splitting. A difficult task is clarifying the chemical dynamics of the carbon structure; the activated species of the water splitting process as well as the electron flow through the carbon-metal interface may well lead to structural changes at the sites of carbon-Pt binding during the operation of the system.

The contribution will elucidate the critical role of modern electron microscopic techniques for understanding and designing functional materials that are critically needed in the challenge of a sustainable energy system.

\section{References:}

[1] R. Schlögl, Angew. Chem. Int. Ed., 50 (2011), 6424.

[2] J.O. Bockris, A. Huq, Proceedings of the Royal Society of London Series a-Mathematical and Physical Sciences, 237 (1956), 277.

[3] C. Galeano, J.C. Meier, V. Peinecke, H. Bongard, I. Katsounaros, A.A. Topalov, A. Lu, K.J.J.

Mayrhofer, F. Schueth, Journal of the American Chemical Society, 134 (2012), 20457. 